\title{
Psychological disturbances in obstetrics (Part II)
}

\author{
Sunera M Fernando ${ }^{1}$ \\ Sri Lanka Journal of Obstetrics and Gynaecology 2009; 31: 125-127
}

Key words: Psychological disturbances in pregnancy

\section{Psychosis in pregnancy}

Research evidence does not identify pregnancy as a period of increased risk for psychosis ${ }^{12}$.

Patients with psychosis especially those with several relapses benefit from continuing antipsychotic medication throughout pregnancy this minimises foetal risks to exposure by reducing the risk of treatment with high doses during a relapse.

\section{Psychopharmacologic treatment in pregnancy}

The goals of treating psychiatric disorders in pregnancy is attaining mental health stability, while minimising risk to the mother and foetus ${ }^{13}$. A number of factors needs to be borne in mind when recommending medication for pregnant women. When prescribing in pregnancy, prescribe only when potential benefits outweigh the potential harm, try to avoid all drugs in first trimester, use established drugs at lowest effective dose, avoid polypharmacy, adjust dose as pregnancy progresses and metabolism alters, ensure adequate foetal screening, be aware of problems with drugs at time of delivery, and monitor infants for withdrawal effects from drugs ${ }^{14}$.

Most women are concerned about prenatal exposure to psychotropic medications. Therefore discussion of the data on medication exposure and teratogenicity versus the potential risk of relapse or symptom exacerbation needs to be done. In other words, the risks of pharmacotherapy must be weighed against the risks of illness relapse since medication and maternal illness can both cause harm to the growing foetus ${ }^{12}$.

In the management of psychiatric disturbance in pregnancy, it is important to discuss the different options for treatment (after reviewing latest available data on drug side effects) with the patient. The patient should actively participate in understanding the possible outcome of treatment and non-treatment and

\footnotetext{
${ }^{1}$ Lecturer, Department of Psychological Medicine, Faculty of Medicine, University of Colombo.

E-mail: sunera@gmail.com
}

making the final treatment choice. During this assessment, the fact that the mental disturbance may impair the women's decision making capacity must be borne in mind. In these circumstances proper documentation is important especially the informed consent of the patient. Each woman must be evaluated individually in collaboration with a psychiatrist, obstetrician and her husband.

Looking at antipsychotic use in pregnancy, most experience is present with chlorpromazine (constipation and sedation can be a problem), trifluoperazine, olanzapine and clozapine (gestational diabetes may be a problems with both these).

Antipsychotic discontinuation symptoms such as crying, agitation, increased suckling can occur in the neonate after delivery ${ }^{14}$.

Considering the use of antidepressants in pregnancy, patients who are already receiving antidepressants and are at high risk of relapse should be maintained on antidepressants during and after pregnancy. Those who develop a depressive episode during pregnancy should be treated with antidepressants if psychological management fails. There is most experience with amitriptyline, imipramine (constipation and sedation are a problem with both), and fluoxetine (increased chance of earlier delivery and reduced birth weight). If the patient is stabilized on another antidepressant the latest evidence should be sought ${ }^{14}$.

In treating bipolar disorder in pregnancy for women who have not had a relapse for a long period, it is possible to withdraw medication. However, for women with relapses mood stabilizer should be advised to continue. No mood stabilizer is clearly safe. Women prescribed lithium should have ultrasound scans at 6 and 18 weeks to look for Ebstein's anomaly (in the foetal heart). Those on valprote or carbamazepine should receive prophylactic folic acid to reduce neural tube defects. Valproate should be avoided as it is the most teratogenic of the mood stabilizers ${ }^{14}$.

\section{Psychiatric disorders in post partum period}

The post partum period is a time of rapid changes (physical and psychological) for the woman. 
Psychiatric disorders that can present during this time are the 'baby blues', postpartum depression and postpartum psychosis.

\section{Baby blues}

Many women experience some affective (mood) symptoms during the postpartum period i.e. 4-6 weeks after delivery. Most of these are the 'baby blues' which are transient mood disturbances with mood lability (changing mood), sadness and tearfulness. These may last a few days and are thought to be due to rapid hormonal changes after delivery, stress of delivery and awareness of the responsibilities of motherhood. Baby blues affects about $25 \%-75 \%$ of new mothers. Education and support for the mother is all that is required for management of this condition. However, if it fails to improve in two weeks then an evaluation for post-partum depression is needed. Women could be educated about these symptoms before delivery and advised to seek medical advice if the symptoms do not improve in two weeks ${ }^{15}$.

\section{Postpartum psychosis}

This is a psychotic disorder that occurs in women after delivery. It affects one in 1000 deliveries. The condition almost always begins within 8 weeks of delivery. The characteristic features are the mother's depression and delusions which are firmly held false beliefs based on incorrect inference about external reality. Delusions are firmly held despite objective contradictory evidence and they are not expected beliefs for the person to have given their education, social and cultural background. The mother can also have thoughts of harming herself or her infant. Such thoughts can be dangerous as some mothers have harmed their infants and themselves. The patient frequently complains of fatigue, insomnia, restlessness, and they have episodes of tearfulness and emotional lability. They also have agitation, suspiciousness, confusion, incoherence, irrational statements, grossly disorganised behaviour that is a considerable change from previous function, and obsessive concerns about the baby's health and welfare. They may have delusions that the baby is dead or malformed. They may even deny the birth. They may have hallucinations (voices) telling the patient to kill the baby or herself.

A careful neurological examination is needed in this condition to exclude other differential diagnoses such as organic neurologic disease i.e.stroke ${ }^{11}$.

Postpartum psychosis is a psychiatric emergency. The mother needs to be monitored carefully. Therefore this condition requires urgent psychiatric referral for assessment and management. The treatment is with antipsychotic medication or Electroconvulsive therapy (ECT).

Subsequent pregnancies are associated with an increased risk of reoccurrence. In women with bipolar affective disorder there is a high risk of post partum psychosis and therefore they need to be monitored for an emerging psychosis in the post partum period.

\section{Postnatal depression}

Postnatal depression is a major public health problem and affects about one in seven women after childbirth $^{8}$. There is a three fold increase in the onset of depression in the first five weeks after birth ${ }^{16}$. It is a serious condition that is generally not self-limiting.

Depressed mood, excessive anxiety, insomnia and change in weight characterises post natal depression which generally occurs within 12 week of delivery.

Apart from the adverse effect on the mother's physical and psychological health, maternal depression also affects the cognitive and emotional development of the infant. Research has shown that it leads to poor care of the infant, unfavourable parenting, discontinuing breast feeding, and even decreased use of preventive services such as infant vaccination $^{11}$.

Post-partum depression is treated with antidepressants and counselling. Current research shows that exercise and omega- 3 fatty acids may also be beneficial ${ }^{17}$.

\section{Psychopharmacologic treatment in the puerperium}

When prescribing medication to women in the post partum period, in each case the benefits of breast feeding to the mother and the infant must be weighed against the risk of drug exposure in the infant. Premature infants and infants with cardiac, renal or hepatic impairment are most at risk from exposure to drugs. The infants should be monitored for specific adverse effects of drugs as well as for feeding pattern and growth and development. Whenever possible use the lowest effective dose and advise the woman to time the feeds to avoid peak drug levels in milk or express milk and give later. The recommended antidepressants during breastfeeding are paroxetimne or sertraline. The antipsychotics recommended are olanzepine or sulpiride. Mood stabilizers should be avoided and if absolutely needed valproate can be used. For sedation in breastfeeding women lorazepam can be used when 
anxiety is also present. Zolpidem can be used to improve sleep in breast feeding women ${ }^{14}$.

\section{Conclusions}

The importance of identifying and managing psychiatric symptoms in women who are pregnant or in the post partum period has been highlighted in this article. These interventions are of great consequence to improve maternal mental well-being which will help her to experience pregnancy as a time of happiness and emotional well being, as well equipping her to provide good care for her newborn.

\section{References}

1. Sadock BJ, Sadock VA. Psychiatry and reproductive medicine. In Kaplan and Sadock's Synopsis of Psychiatry. 10th ed. 2007, New York: Wolters Kluwer/Lippincott Williams \& Wilkins.

2. Gelder M, Harrison P, Cowen P. Psychiatric aspects of medical procedures and conditions. In Shorter Oxford Textbook of Psychiatry, 2006, Oxford: Oxford University Press.

3. Hughes P, Riches S. Psychological aspects of perinatal loss. Current Opinion in Obstetrics and Gynaecology 2003; 15: $107-11$.

4. Gotleib IH, Whiffen VE, Mount JH, Milne K, Cordy NL. Prevalence rates and demographic characteristics associated with depression in pregnancy and the postpartum. Journal of Consulting Clinical Psychology 1989; 57: 269-74.

5. Nonacs R, Cohen LS. Deprression during pregnancy: diagnosis and treatment options. Journal of Clinical Psychiatry 2002; 63(Suppl 7): 24-30.

6. Altshuler LL, Hendrick V, Cohen LS. Course of mood and anxiety disorders during pregnancy and the post partum period. Journal of Clinical Psychiatry 1998; 59(Suppl. 2): 29-33.

7. Zuckerman B, Amaro H, Bauchner H, Cabral H. Depressive symptoms during pregnancy: relationship to poor health behaviours. American Journal of Obstetrics and Gynaecology 1989; 60: 1107-11.

8. Lumley J, Austin M. What interventions may reduce postpartum depression. Current Opinion in Obstetrics and Gynaecology 2001; 13: 605-11.

9. Orr ST, Miller CA. Maternal depressive symptoms and the risk of poor pregnancy outcome. Epidemiology Review 1995; 17: $165-71$.

10. Allister L, Lester BM, Carr S, Liu J. The effects of maternal depression on fetal heart response to vibroacoustic stimulation. Developmental Neuropsychology 2001; 20(3): 639-51.

11. Tam WH, Cheung T. Psychosomatic disorders in pregnancy. Current Opinion in Obstetrics and Gynaecology 2007; 19: 126-32.

12. Cott AD, Wisner KL. Psychiatric disorders during pregnancy. International Review of Psychiatry 2003; 15: 217-30.

13. Zisook S, Burt V K. Psychiatric disorders during pregnancy. Psychiatric Times 2003; 20(1):

14. Maudsley Prescribing Guidelines 2007-2008. The South London and Maudsley Trust. pp. 268-82.

15. Sadock B, Sadock VA. Psychiatry and reproductive medicine. In Kaplan and Sadock's Concise Textbook of Clinical Psychiatry. 3rd ed. 2008, New York: Wolters Kluwer / Lippincott Williams \& Wilkins.

16. Cox JI, Murray D, Chapman G. A controlled study on the onset, duration and prevalence of postnatal depression. British Journal of Psychiatry 1993; 163: 27-31.

17. Shaw E, Kaczorowski J. Postpartum care - what's new? Current Opinion in Obstetrics and Gynaecology 2007; 19: 561-7. 